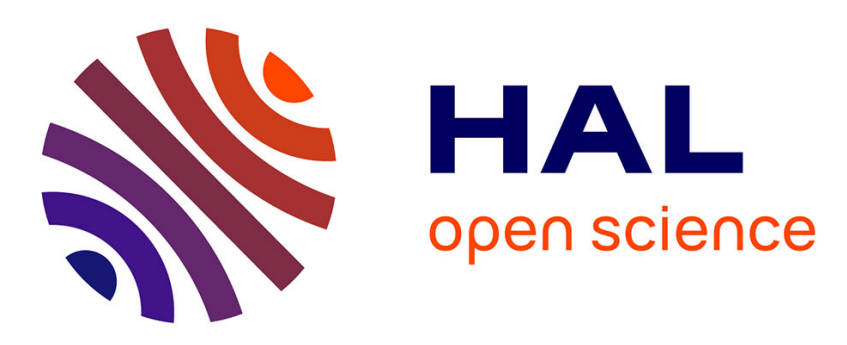

\title{
A New Method to Measure 3D Textile Defects by Using Dual-lens Camera
}

\author{
Jun Xu, Christophe Cudel, Sophie Kohler, Stéphane Fontaine, Olivier \\ Haeberlé, Marie-Louise Klotz
}

\section{- To cite this version: \\ Jun Xu, Christophe Cudel, Sophie Kohler, Stéphane Fontaine, Olivier Haeberlé, et al.. A New Method to Measure 3D Textile Defects by Using Dual-lens Camera. Advanced Materials Research, 2013, 821 - 822, pp.237-242. 10.4028/www.scientific.net/AMR.821-822.237 . hal-00984503}

\section{HAL Id: hal-00984503 https://hal.science/hal-00984503}

Submitted on 28 Apr 2014

HAL is a multi-disciplinary open access archive for the deposit and dissemination of scientific research documents, whether they are published or not. The documents may come from teaching and research institutions in France or abroad, or from public or private research centers.
L'archive ouverte pluridisciplinaire HAL, est destinée au dépôt et à la diffusion de documents scientifiques de niveau recherche, publiés ou non, émanant des établissements d'enseignement et de recherche français ou étrangers, des laboratoires publics ou privés. 


\title{
A New Method to Measure 3D Textile Defects by Using Dual-lens Camera
} Jun.Xu ${ }^{123, a}$, Christophe.Cudel ${ }^{2, b}$, Sophie.Kohler ${ }^{2, c}$, Stephane.Fontaine ${ }^{4, d}$, Oliver.Haeberle $^{2, e}$, Marie-Louise.Klotz ${ }^{1, f}$

\author{
${ }^{1}$ Rhine-Waal University of Applied Sciences, Marie-Curie-Strasse 1, 47533, Kleve Germany \\ ${ }^{2}$ Laboratoire Modélisation Intelligence Processus Systèmes MIPS, EA 2332, Université de Haute \\ Alsace, 61 rue Albert Camus, 68093 Mulhouse Cedex France \\ ${ }^{3}$ The Research Institute for Textile and Clothing, Niederrhein University of Applied Sciences, \\ Webschulstr. 31, 41065 Moenchengladbach Gemany \\ ${ }^{4}$ ISAT Laboratory, EA 1859, Université de Bourgogne, 49, rue Mademoiselle Bourgeois, 58027 \\ Nevers France \\ a jun.xu@hochschule-rhein-waal.de \\ bchristophe.cudel@uha.fr \\ ${ }^{\mathrm{c}}$ sophie.kohler@uha.fr \\ d Stephane.Fontaine@u-bourgogne.fr \\ e olivier.haeberle@uha.fr \\ f marie-louise.klotz@hochschule-rhein-waal.de
}

Keywords: textile defect, homography, stereovision, computer vision, glass fiber fabric, nonwoven

\begin{abstract}
Industrial textile fabric with functionalization always has a high standard requirement. Take polytetrafluoroethylene (PTFE) conveyor belt as an example, the base fabrics must be of first-rate quality, with no weaving faults or broken fibers on coated surface [1]. In a previous work [2], we have proposed an original method to measure the height of the fiber based on variable homography. This measurement is based on a single camera acquiring two successive frames. This scheme is working well, but measurement depends on conveyor speed used for scrolling fabric. In this paper, we propose an improvement by using a new acquisition device based on two mini-lenses assembled as a dual-objective. With this device, variable homography modeling leads to 3-D online inspection whatever fabric speed with a simplified calibration procedure compared to classic stereovision approaches.
\end{abstract}

\section{Introduction}

Variable homography has been introduced by Zhang and Greenspan [3] to improve image mosaicking, in order to overcome parallax between objects located at different depths. We have extended the concept of variable homography $[2,4]$ for 3-D measurements. We have applied this model to measure emergent fibers on glass and non-woven fabrics, and we have validated the interest of this method. Even if uncertainty of measurement is lower than $10 \%$, measurement quality would depend on the conveyor speed. To avoid being dependent of this speed, we have modified the acquisition setup by using two mini-lenses assembled in one camera. The idea is to obtain two projected images on a single CCD sensor. There are some advantages to model this tiny stereovision system with variable homography instead of simple triangulation as done most of time. Each projection system has its own intrinsic parameters, which are easy to integrate with variable homography modeling and calibration stage is simplified. Experimental results prove uncertainty of measurement to be lower than 5\% on emergent fibers having a diameter of $100 \mu \mathrm{m}$ and being 1 to 5 $\mathrm{mm}$ long. At this scale, this dual-lens setup fills the gap range between microscopy $(\mu \mathrm{m}-\mathrm{mm})$ and 3-D vision (cm-m) to perform 3-D measurements online. 


\section{Variable Homography}

Fig. 1(a) give a representation of our system made out of 2 mini-lenses and a single image plane. This scheme corresponds to a parallel stereovision system, and a simple triangulation permits to compute $Z_{B} . Z_{B}$ is then formulated with the $p_{L B}\left(u_{L B}, v_{L B}\right)$ and $p_{R B}\left(u_{R B}, v_{R B}\right)$ coordinates, the lens focal $f$ and distance $b_{\mathrm{x}}$ between lens optical centers:

$$
Z_{B}=f \cdot b_{x} /\left(u_{L B}-u_{R B}\right)
$$

This relation works well if camera centers and focals are identical for both image projections. In experimental conditions these intrinsic parameters are never strictly identical. Eq. (1) can be adapted to take them into account, but at this scale of measurement, a manual unfriendly calibration is necessary for their determination. Variable homography could be an alternate to simplify the calibration step.

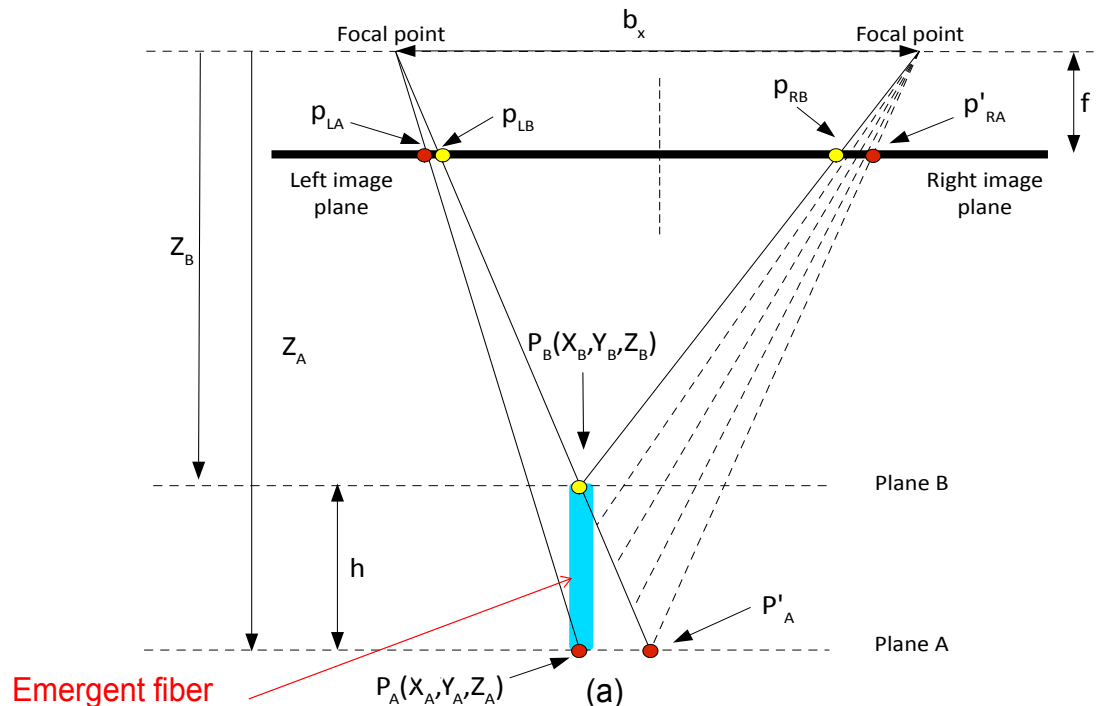

(b)

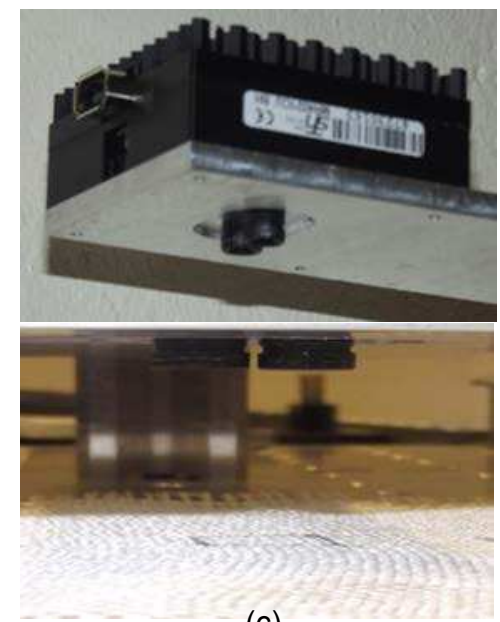

(c)

Figure 1. (a) configuration of dual-lens device, views of experimental prototype. (b) the dual-lens device; (c) image acquisition of a glass fiber fabric

\section{Principle of Variable Homography}

When two points have same $X$ and $Y$ coordinates, as points $P_{A}\left(X_{A}, Y_{A}, Z_{A}\right)$ and $P_{B}\left(X_{B}, Y_{B}, Z_{B}\right)$ on Fig. $1(\mathrm{a})$, the first result of variable homography gives a relation between their projections $\mathrm{p}_{\mathrm{LA}}$ and $\mathrm{p}_{\mathrm{LB}}$ onto the image plane:

$$
p_{L B}=\left(\begin{array}{c}
u_{L B} \\
v_{L B} \\
1
\end{array}\right)=\left(\begin{array}{ccc}
k & 0 & (1-k) u_{0 L} \\
0 & k & (1-k) v_{0 L} \\
0 & 0 & 1
\end{array}\right)\left(\begin{array}{c}
u_{L A} \\
v_{L B} \\
1
\end{array}\right)=K \cdot p_{L A}
$$

where $\mathrm{k}=\mathrm{Z}_{\mathrm{A}} / \mathrm{Z}_{\mathrm{B}}$ and $\left(\mathrm{u}_{0}, \mathrm{v}_{0}\right)$ are the coordinates of focal point projection on image plane. By adding a second camera [3], one can define homographies $\mathrm{H}_{\mathrm{A}}$ and $\mathrm{H}_{\mathrm{B}}$, linking image projections of points contained within planes A and B, respectively:

$$
p_{R A}=\left(\begin{array}{c}
u_{R A} \\
v_{R A} \\
1
\end{array}\right)=H_{A}\left(\begin{array}{c}
u_{L A} \\
v_{L A} \\
1
\end{array}\right)=H_{A} \cdot p_{L A} \text { and } p_{R B}=\left(\begin{array}{c}
u_{R B} \\
v_{R B} \\
1
\end{array}\right)=H_{B}\left(\begin{array}{c}
u_{L B} \\
v_{L B} \\
1
\end{array}\right)=H_{B} \cdot p_{L B}
$$

We consider plane $\mathrm{A}$ as a reference plane. If plane $\mathrm{B}$ is parallel to plane $\mathrm{A}$ and located at a variable height, the corresponding homography, noted $\mathrm{H}_{\mathrm{B}}(\mathrm{k})$, can be deduced from $\mathrm{H}_{\mathrm{A}}$ and $\mathrm{K}$ :

$$
H_{B}(k)=K \cdot H_{A} \cdot K^{-1}
$$




\section{Measurement Principle}

For any emergent fiber having an intersection with the virtual plane $\mathrm{B}$, we propose to measure the height of plane B through the horizontal distance between the projections $p^{\prime}{ }_{R A}$ and $p_{R B}$. From Eq. (4) and analytic expression of homography $\mathrm{H}_{\mathrm{A}}[5]$, this distance is given by:

$$
d_{u}(k)=u_{R A}^{\prime}-u_{R B}=(k-1) \cdot \frac{b_{x} \cdot f}{Z_{A}}=(k-1) \cdot C_{u}
$$

where $b_{x}$ is the distance between the focal points and $f$ the lens focal distance. During a measurement, $\mathrm{d}_{\mathrm{u}}(\mathrm{k})$ is proportional to $(\mathrm{k}-1)$, and plane $\mathrm{B}$ height, i.e. fiber height, is calculated with:

$$
h=Z_{A}-Z_{B}=Z_{A}-\frac{Z_{A}}{k}=Z_{A} \frac{d_{u}(k)}{d_{u}(k)+C_{u}}
$$

The measurement process can be resumed in four steps, as illustrated on Fig. 2:

- Step 1 (left image): detection of point belonging to a fiber, noted $\mathrm{p}_{\mathrm{LB}}$;

- Step 2: computation of $\mathrm{p}_{\mathrm{RA}}$ according: $\mathrm{p}^{\prime}{ }_{\mathrm{RA}}=\mathrm{H}_{\mathrm{A}} \cdot \mathrm{p}_{\mathrm{LB}}$;

- Step 3 (right image): detection of corresponding $p_{L B}$ point belonging to the fiber, noted $p_{R B}$;

- Step 4: computation of $h$ from $d_{u}$.

This height measurement is depending on $\mathrm{H}_{\mathrm{A}}, \mathrm{C}_{\mathrm{u}}$ and $\mathrm{Z}_{\mathrm{A}}$. These three parameters can be determined by a simple calibration described in section Calibration.

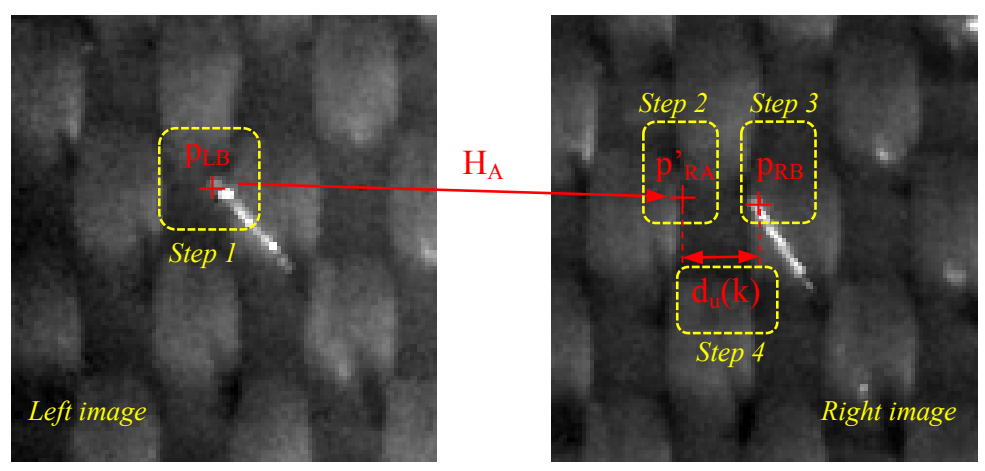

Figure 2. Measurement process in four steps

\section{Calibration}

We propose a simple, two-step calibration process realized through chessboard acquisitions. The first acquisition is performed at the reference height $Z_{\mathrm{A}}$. The second one is done by elevating the pattern by $\mathrm{dz}$. This second acquisition is then performed at height $\mathrm{Z}_{\mathrm{B}}=\mathrm{Z}_{\mathrm{A}}-\mathrm{dz}$ : $\mathrm{k}$ is therefore known.

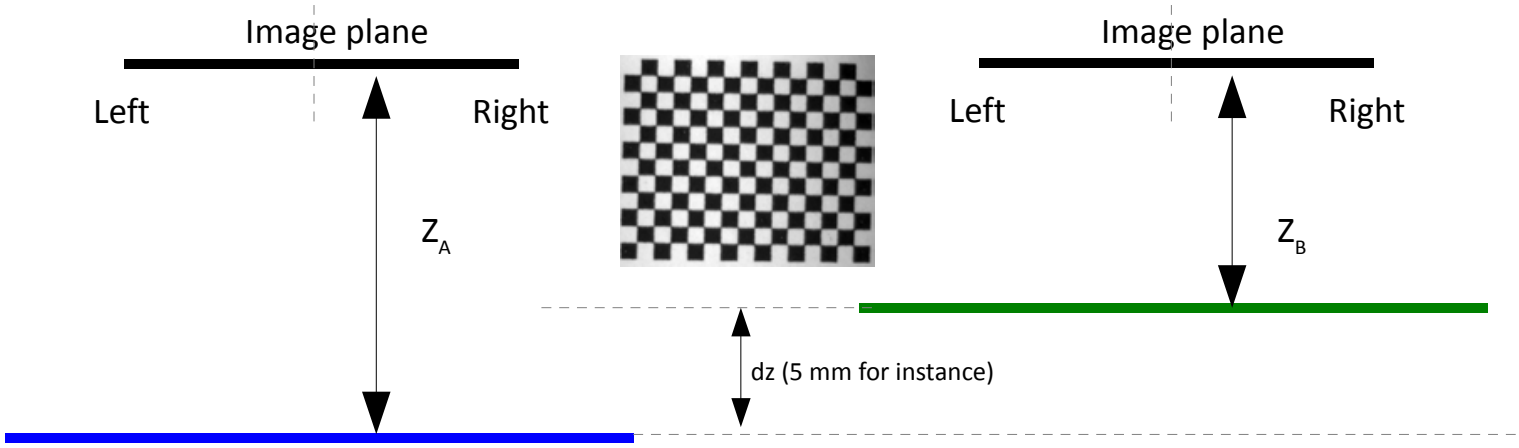

Figure 3. Calibration in two steps: reference plane acquisition (plane $A$ ); acquisition at height $Z_{B}$ 


\section{$1^{\text {st }}$ acquisition: $\mathrm{H}_{\mathrm{A}}$}

Chessboard corners are detected on both images and are used to calculate homography $\mathrm{H}_{\mathrm{A}}$. We note $\mathrm{d}_{\mathrm{A}}$ the distance in pixels between the top-left and bottom-right corners.

\section{$2^{\text {nd }}$ acquisition: $Z_{A}$ and $C_{u}$}

The second set of chessboard corners is detected. We note $d_{B}$ the distance in pixels between the top-left and bottom-right corners. $\mathrm{Z}_{\mathrm{A}}$ is obtained by triangulation and can be formulated as:

$$
Z_{A}=\frac{d_{Z} \cdot d_{B}}{d_{B}-d_{A}}
$$

This second acquisition is also used to estimate the constant $\mathrm{C}_{\mathrm{u}}$. Corners detected on both images constitute a dataset of $\mathrm{p}_{\mathrm{LB}}$ and $\mathrm{p}_{\mathrm{RB}}$ points. By using $\mathrm{H}_{\mathrm{A}}$ computed at the previous step, the correspondences $\mathrm{p}_{\mathrm{RA}}$ are then computed and $\mathrm{C}_{\mathrm{u}}$ is calculated from Eq. (5). This calibration method is well adapted for 3-D online measurement. Compared to a calibration process used to determine intrinsic parameters [6], our method is easier to perform and can be executed without difficulty by an operator.

\section{Experimental Results}

In this part, we first evaluate the accuracy of our method and then present some results on textile fabrics.

\section{Measurement performance}

To evaluate performance and accuracy, we use as reference pattern a pyramidal object with five levels, each separated by $1 \mathrm{~mm}$. Fig. 4 presents a typical measurement for each step when matching between $p_{L B}$ and $p_{R B}$ points is precise.

On this example, measurement error is below $1 \%$. We must be careful with this excellent result because accuracy is strongly dependent of the matching process. With this pyramidal object and by using calibration parameters, it's possible to determine theoretical position of each correspondence by fixing $\mathrm{k}$ at $\mathrm{Z}_{\mathrm{A}} /\left(\mathrm{Z}_{\mathrm{A}}-\mathrm{h}\right)$, according to the selected level. On Fig. 5a, we have zoomed on two selected points in left image and located respectively at 3 and $4 \mathrm{~mm}$ elevation. Fig. $5 \mathrm{~b}$ shows the corresponding right image, where the theoretical matching positions are represented with red crosses and selected positions for measurements with blue ones. At $3 \mathrm{~mm}$ level position, red and blue crosses are close and measurement based on the blue point gives a height of $3.08 \mathrm{~mm}$. At $4 \mathrm{~mm}$, we have forced a shifting of more than 3 pixels between theoretical position and measurement position. In this case the measured height is $3.56 \mathrm{~mm}$ instead of $4 \mathrm{~mm}$. This example shows the importance of the correspondence matching process. With calibration parameters values, we can evaluate the influence of matching process. The uncertainty measurement is essentially function of $\mathrm{d}_{\mathrm{u}}(\mathrm{k})$ and can then be formulated from Eq. (6):

$$
\frac{\partial h}{\partial\left(d_{u}(k)\right)}=Z_{A} \cdot \frac{C_{u}}{\left(d_{u}(k)+C_{u}\right)^{2}}
$$

On this example, the calibration process gives $\mathrm{Z}_{\mathrm{A}}=3.81 \mathrm{~cm}$ and $\mathrm{C}_{\mathrm{u}}=-234$ pixels. The uncertainty measurement on $\mathrm{h}$ is then $0.16 \mathrm{~mm}$ per pixel. Therefore, following the nature of the samples to be measured, the matching method must be adapted. By using sub-pixel matching algorithms ensuring a matching of \pm 0.25 pixel, the uncertainty measurement is lower than $\pm 0.04 \mathrm{~mm}$ or $4 \%$ for a $1 \mathrm{~mm}$ height fiber. 


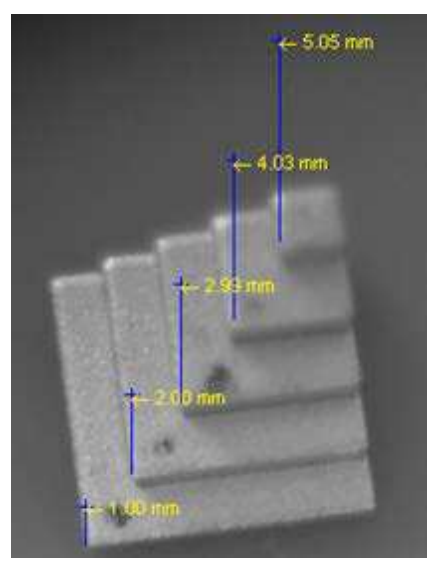

Figure 4. Measurements on a reference pyramidal object
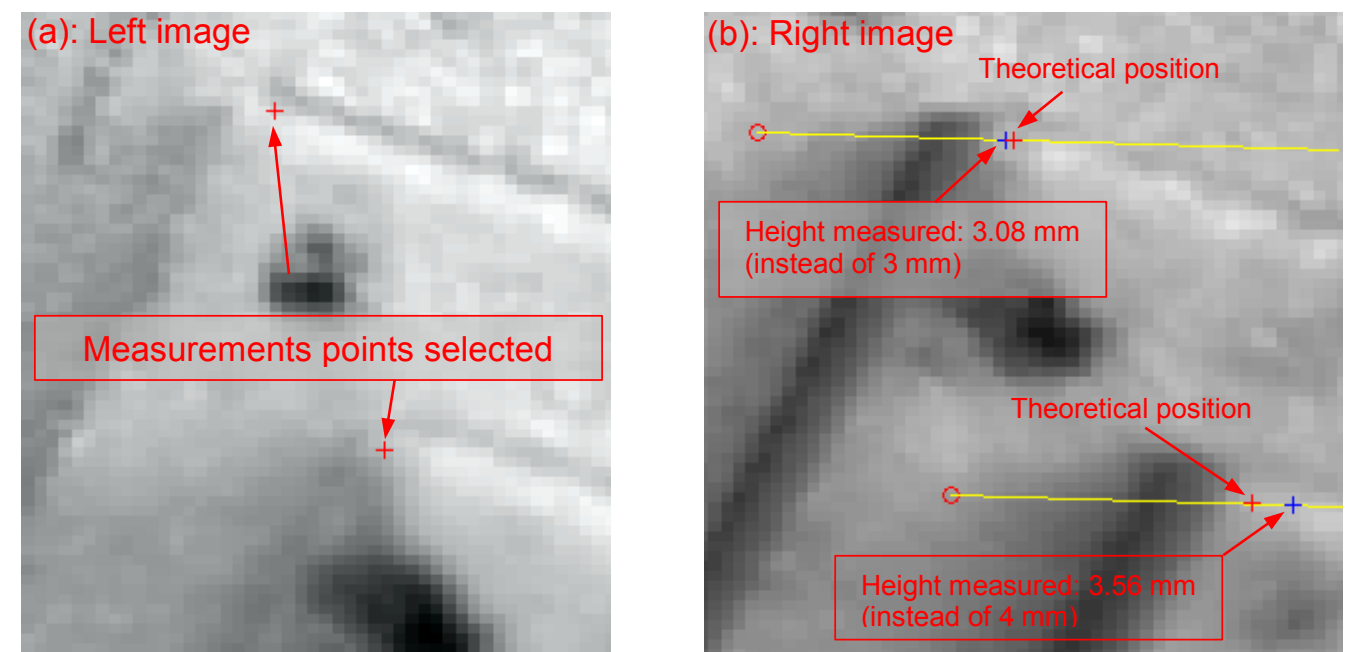

Figure 5. Zoom on matching points between left and right images. On right image, red crosses are positioned on theoretical positions of measurement points selected in left image. The blue crosses are positions used for measurements. Heights measured are strongly dependent on the matching process

\section{Measurements on Textile Fabrics}

This part presents some results obtained on textile fabrics to evaluate the height of emergent fibers. We use a laser line illumination to ensure good correspondences. This illumination skims the fabric surface. Interest for this application is to only highlight emergent fibers. From bright spots on left images, correspondences are searched in right images along the line (so-called epipolar line [2]) describing the theoretical positions for various $\mathrm{k}$ values (depicted as yellow lines visible on Fig. 5b). It is then possible to select a set of points on the emergent fiber, and then compute its corresponding 3-D profile. Fig. 6 gives a first result on an analysis surface sizing approximately $4 \mathrm{~cm}^{2}$. This measurement is done on a glass fabric presenting a broken fiber. Fiber stands straight, which leads to the computed profile on the zoomed region. Other typical examples on glass and non-woven fabrics are presented on Fig. 7. 


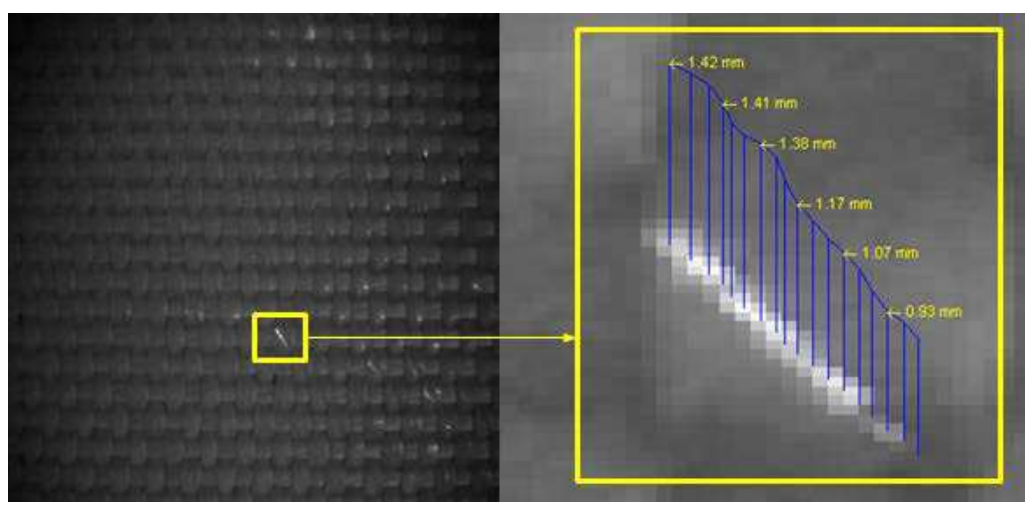

Figure 6. Measurements of emergent glass fabric fibers
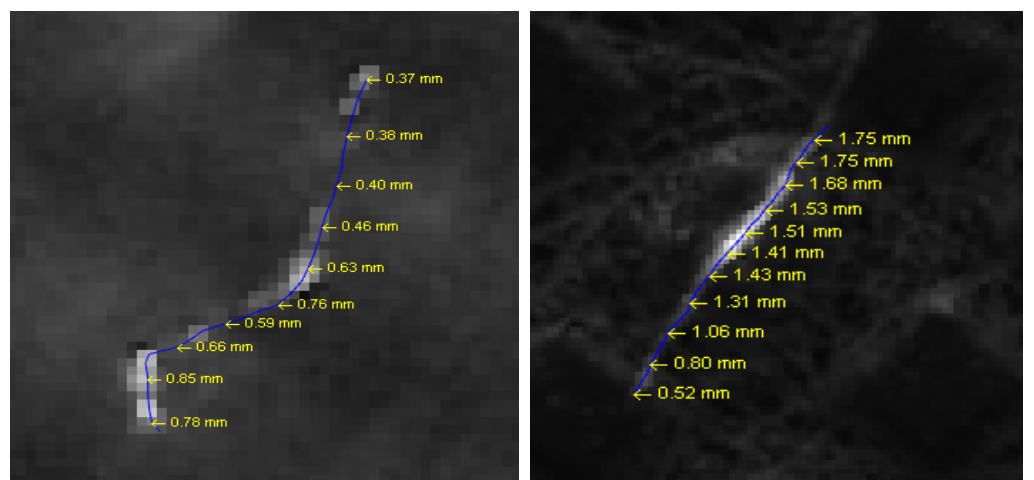

Figure 7. Example of profiles on glass and non-woven fabric

\section{Conclusion}

This work proposes an original 3-D measurement approach to inspect glass and non-woven fabrics. Variable homography modeling allows for a simplified calibration process, which can easily be executed by an operator. The measurement principle has been validated on a reference pyramidal object. In our experimental acquisition conditions, we estimate the uncertainty measurement to stay below 5\%. This method can be extended to other applications, the matching method being to be adapted to the characteristics of the images. At this scale, most of measurement processes need to sample part of glass fabric and realize offline measurements with a laboratory equipment. Our device with two associated mini-lenses and with this original measurement method should be able to work online in an industrial environment, and could be extended to a complete online 3-D surface fabric reconstruction.

\section{References}

[1] W. Fung: Coated and Laminated textiles, CRC Press, ISBN 1855735768, Cambridge(UK), (2002)

[2] J. Xu, C. Cudel, S. Kohler, S. Fontaine, O. Haeberlé, and M.-L. Klotz: Original method to compute epipoles using variable homography: application to measure emergent fibers on textile fabrics, in Journal of Electronic Imaging, Vol. 21 (2012), N 2 2, pp. 021103-1-021103-11

[3] S. Zhang and M. Greenspan: Variable Homography Compensation of Parallax Along Mosaic Seams, Lecture notes in computer science, Vol. 4663 (2007), pp. 271-284, ISSN 0302-9743

[4] J. Xu, C. Cudel, S. Kohler, S. Fontaine, O. Haeberlé, and M.-L. Klotz: Textile emergent fibers length measurement system based on camera vision and variable homography, 11th World Textile Conference, Proceedings of AUTEX, ISBN 978-2-7466-2858-8, Mulhouse, June, (2011)

[5] J. Xu: Using variable homography to measure protruding fibers on textile fabrics, $\mathrm{PhD}$ dissertation, July 2012, Université de Haute Alsace, Mulhouse, France

[6] Z. Zhang: A flexible new technique for camera calibration, IEEE transactions on pattern and machine intelligence, Vol. 22 (2012), $\mathrm{N}^{\circ} 11$, pp. 1330-1334 